JR Parkinson, MJ Hyde, C Gale, S Santhakumaran, N Modi. Neonatology, Imperial College, London, UK

Background and Aims Preterm birth is associated with raised blood pressure (BP) and other features of the metabolic syndrome in later life, but effect sizes and biological mechanisms are unknown. We conducted a meta-analysis to address these associations in adult life.

Methods We performed a systematic review and meta-analysis of studies in which metabolic syndrome associated indices were compared in adults ( $\geq 18$ years of age) born preterm $(<37$ weeks gestation) and at term (37-42 weeks gestation). Outcome measures included; systolic blood pressure (SBP), diastolic blood pressure (DBP), BMI, percentage fat mass and fasting plasma levels of lipids, glucose and insulin.

Results Data from 27 studies and 306,123 adults (16,094 preterm, 290,029 term) were included, with an average outcome age of 26.1 years. In adults, preterm compared with full-term birth was associated with significantly higher SBP (mean difference [95\% confidence interval]: $4.2 \mathrm{mmHg}[2.7,5.7], \mathrm{p}<0.001), \mathrm{DBP}(2.7 \mathrm{mmHg}[1.2,4.2]$, $\mathrm{p}<0.001)$ and low density lipoprotein (LDL) $(0.14 \mathrm{mmol} / \mathrm{L}[0.05$, 0.22 ], $\mathrm{p}=0.01$ ). Meta-regression revealed a significant gender effect, with $3.0 \mathrm{mmHg}$ greater SBP in preterm compared to term women than in preterm-term men ( $95 \% \mathrm{CI}: 1.3,4.7, \mathrm{p}=0.002)$; for DBP this difference was $2.1 \mathrm{mmHg}$ greater $(0.6,3.6, \mathrm{p}=0.009)$.

Conclusions Preterm compared to term birth, is associated with higher blood pressure and LDL in adult life. Women born preterm appear to be at greater risk than men born preterm. Follow-up of older subjects born preterm will be required to determine if the effects we observe are exacerbated by age.

\section{ADIPOCYTOKINES OMENTIN AND VASPIN ARE UPREGULATED IN LARGE FOR GESTATIONAL AGE FETUSES AT TERM}

doi:10.1136/archdischild-2012-302724.0354

${ }^{1} \mathrm{G}$ Kafalidis, 'DD Briana, 'T Boutsikou, 'M Boutsikou, ${ }^{2} \mathrm{~A}$ Marmarinos, ${ }^{1} \mathrm{~S}$ Baka, ${ }^{1} \mathrm{D}$ Hassiakos, ${ }^{2} \mathrm{D}$ Gourgiotis, 'A Malamitsi-Puchner. 'Neonatal Division; 2nd Department of Obstetrics and Gynecology; ${ }^{2}$ Research Laboratory of Clinical Biochemistry-Molecular Diagnostics; 2nd Department of Pediatrics, Athens University Medical School, Athens, Greece

Background and Aims Fetal macrosomia is associated with significant perinatal and long-term complications, including higher risk for later development of insulin resistance/metabolic syndrome. Besides regulating whole body metabolism, adipocytokines have been implicated in fetal growth. We aimed to investigate circulating concentrations of omentin-1 and vaspin (important adipocytokines, regulating glucose metabolism and insulin sensitivity) in fetal samples from large-for-gestational-age-(LGA) and appropriate-forgestational-age-(AGA) pregnancies and correlate them with several maternal and fetal anthropometric/clinical variables.

Methods Sixty five LGA (14 born from mothers presenting with gestational diabetes mellitus and 51 born from non-diabetic mothers) and 35 AGA singleton full-term infants were recruited. Determination of cord blood omentin-1 and vaspin concentrations was performed by enzyme-linked immunosorbent assay.

Results Cord blood omentin-1 concentrations were significantly higher in LGA compared to AGA neonates $(b=0.119, p=0.002$, SE 0.036), after controlling for confounding factors. Similarly, cord blood vaspin concentrations were significantly elevated in LGA cases, compared to AGA controls $(p=0.011)$. Finally, cord blood omentin-1 concentrations were lower in cases of vaginal delivery ( $b=0.072, p=0.020$, SE 0.030), after controlling for group.

Conclusions Higher concentrations of omentin-1 and vaspin in LGA compared to AGA fetuses, probably suggest the potential role of both adipocykines in intrauterine growth, as well as their possible implication in the metabolic disturbances characterizing fetal macrosomia both in the short- and long-term. Vaginal deliveryassociated inflammation may account for the lower cord blood omentin-1 concentrations.

\section{FETAL GROWTH IS ASSOCIATED WITH ALTERED EXPRESSION OF IMPRINTED GENES IN THE PLACENTA}

doi:10.1136/archdischild-2012-302724.0355

${ }^{1,2} \mathrm{C}$ Piyasena, 'B Khulan, ${ }^{2} \mathrm{G}$ Menon, ${ }^{1} \mathrm{AJ}$ Drake. 'Department of Endocrinology, Centre for Cardiovascular Science, Queen's Medical Research Institute, University of Edinburgh ${ }^{2}$ Simpson Centre for Reproductive Health, Royal Infirmary of Edinburgh, Edinburgh, UK

Background and Aims Both low and high birthweight is associated with adverse health outcomes throughout life. Altered expression of imprinted genes which regulate fetal and placental growth may be one mechanism linking the environment and later disease risk. We have studied the expression of candidate imprinted genes in placenta with respect to anthropometric parameters at birth.

Methods 58 term placentas (27 male) were obtained from the Edinburgh Reproductive Tissue BioBank. Pregnancies complicated by congenital abnormalities or diabetes were excluded. Gene expression was analysed using real-time PCR.

Results Median birthweight was $3900 \mathrm{~g}$ (interquartile range: 2949-4340g). Insulin-like growth factor 2 (IGF2) mRNA levels correlated positively with standard deviation scores for birthweight (Spearman's rho $=0.335, p=0.005$ ), head circumference (Spearman's rho $=0.424, p=0.001$ ) and length (Spearman's rho $=0.259, p=0.041$ ). Growth factor receptor-bound protein 10 (GRB10) mRNA levels correlated negatively with birthweight standard deviation score (Spearman's rho $=-0.221, p=0.048$ ). The expression of two other imprinted genes, PHLDA2 and ZIM2 showed no relation to size at birth.

Conclusion Both IGF2 and GRB10 are imprinted in the placenta and impact on fetal and placental growth. IGF2 is paternally imprinted and increased expression is implicated in overgrowth disorders; in contrast, GRB10 is maternally imprinted in trophoblasts and disruption in mice leads to overgrowth. Additionally, GRB10 has recently been identified as having a role in insulin signaling. As genomic imprinting is under epigenetic regulation, these targets are strong candidates for exploration of environmentally influenced non-Mendelian effects on fetal size and developmental programming.

\section{ALBUMIN SYNTHESIS RATES IN VLBW INFANTS - EFFECTS OF HIGH DOSE AMINO ACID AND LIPID ADMINISTRATION FROM BIRTH ONWARDS}

doi:10.1136/archdischild-2012-302724.0356

${ }^{1} \mathrm{H}$ Vlaardingerbroek, ${ }^{1} \mathrm{D}$ Rook, ${ }^{1,2 \mathrm{H}}$ Schierbeek, ${ }^{3} \mathrm{CHP}$ van den Akker, 'MJ Vermeulen, ${ }^{2,4} \mathrm{JB}$ van Goudoever. 'Pediatrics, Division of Neonatology, Erasmus MC - Sophia Children's Hospital, Rotterdam; 'Pediatrics, Academic Medical Center - Emma Children's Hospital, Amsterdam; ${ }^{3}$ Pediatrics, Erasmus MC - Sophia Children's Hospital, Rotterdam; ${ }^{4}$ Pediatrics, VU University Medical Center, Amsterdam, The Netherlands

Introduction Albumin is one of the most important proteins in plasma and plays a key role in physiological processes like preservation of colloid osmotic pressure and binding of bilirubin and drugs. However, albumin concentrations are often low during the first days of life in preterm infants.

We hypothesized that early parenteral lipid and high dose amino acid (AA) administration from birth onwards to very low birth weight (VLBW) infants increases hepatic albumin synthesis rates and albumin concentration.

Methods Inborn VLBW infants were randomized to one of three different parenteral nutritional regimens within 6hrs after birth 
(Figure 1). Lipids were started at $2 \mathrm{~g} /(\mathrm{kg} . \mathrm{d})$ and increased to $3 \mathrm{~g} /$ (kg.d) the following day.

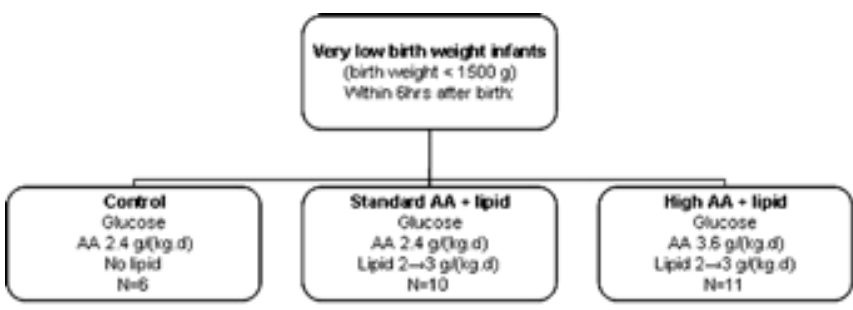

Abstract 356 Figure 1 Study design

At postnatal day 2, infants received a primed continuous infusion of $\left[{ }^{13} \mathrm{C}_{6},{ }^{15} \mathrm{~N}\right]$ leucine. Mass spectrometry was used to determine the fractional and absolute albumin synthesis rates (FSR and ASR, respectively).

Results Albumin FSR, concentration, and ASR were not significantly different between groups (Figure 2; median, IOR).
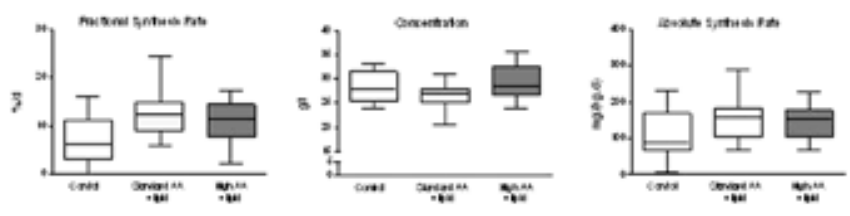

Abstract 356 Figure 2 Albumin FSR, concentration, and ASR

Conclusion Albumin concentration was in normal range in all groups. Albumin synthesis rates and concentration are not increased upon introduction of $2 \mathrm{~g}$ lipids/(kg.d) and 3.6g AA/ $(\mathrm{kg} . \mathrm{d})$ from birth onwards.

\section{HUMAN MILK BILE SALT-STIMULATED LIPASE: THE DYNAMIC CHANGES DURING LACTATION}

doi:10.1136/archdischild-2012-302724.0357

'P Piemontese, ${ }^{2} \mathrm{P}$ Roncada, ${ }^{3} \mathrm{~A}$ Soggiu, ${ }^{3} \mathrm{~L}$ Bonizzi, ${ }^{4} \mathrm{~A}$ Budelli, ${ }^{5} \mathrm{C}$ Agostoni, ${ }^{\mathrm{M}} \mathrm{ML}$ Giannì, IP Roggero, 'F Mosca. 'Department of Maternal and Pediatric Sciences, NICU Fondazione IRCCS 'Ca' Granda' Ospedale Maggiore Policlinico, University of Milan; ${ }^{2}$ Istituto Sperimentale Italiano Lazzaro Spallanzani; ${ }^{3}$ DIPAV, University of Milan; ${ }^{4} \mathrm{Heinz}$ Italia Spa; ${ }^{5}$ nstitute of Pediatrics, University of Milan, Fondazione IRCCS 'Ca' Granda' Ospedale Maggiore Policlinico Milan, Milan, Italy

Background and Aims The efficient digestion of milk triglycerides is guaranteed by the combined action of gastric lipase, colipasedependent pancreatic lipase, and bile salt-stimulated lipase (BSSL). Human milk contains the major part of BSSL. Data regarding the dynamic changes of human milk BSSL are scarce.

The aim of this study was to evaluate the changes of human milk BSSL content at different stages of lactation by proteomic techniques.

Methods We designed a prospective longitudinal study. Human milk samples were collected from 18 healthy mothers who delivered a singleton term newborn (Gestational age 37-41 weeks) on the $3^{\text {th }}$, $7^{\text {th }}, 15^{\text {th }}$, and $60^{\text {th }}$ postpartum day. Proteomic techniques were carried out to evaluate different profile expression of BSSL in milk: in particular SDS-PAGE analysis coupled with LC-MS MS mass spectrometry.

Results BSSL levels increased significantly from the $3^{\text {th }}$ to the $60^{\text {th }}$ postpartum day (figure).

\section{human milk BSSL}

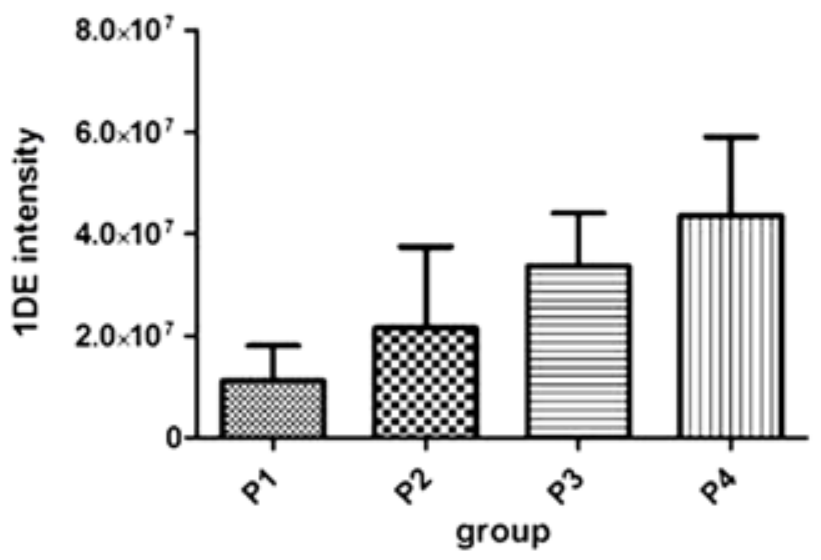

Abstract 357 Figure 1 Human milk BSSL at different study points

Conclusions The increased levels of human milk BSSL could compensate for low endogenous capacity to digest dietary fat in early life as efficient absorption of lipids is important, not only for energy utilization but also for optimal growth and functional neurodevelopment.

\section{THE EFFECT OF GALACTAGOGUE HERBAL TEA ON OXIDANT AND ANTI-OXIDANT STATUS OF HUMAN MILK}

doi:10.1136/archdischild-2012-302724.0358

S Kavurt, AY Bas, 0 Aydemir, H Yücel, N Demirel. Neonatology, Etlik Zübeyde Hanım Women's Health Teaching and Research Hospital, Ankara, Turkey

Background and Aims Human milk (HM) has a number of antioxidant constituents which may protect newborns against oxidative damage. Poor HM production is the most frequent cause of breastfeeding failure. Galactagogues are believed to stimulate initiation and maintenance of HM production. Herbal tea containing fenugreek has recently been presented in the market. We aimed to compare global oxidant and antioxidant capacity of HM among mothers drinking galactagogue herbal tea or not.

Methods Volunteer mothers 18-35 years of age without any antenatal or perinatal risk factors. Milk samples were collected in the first day after delivery. Mothers were randomly assigned to receive galactagogue herbal tea (Humana still-tee $\left.{ }^{\circledR}\right) 3$ cups/day or same amount of water as placebo. Second samples were obtained 7-10 days later. The total antioxidant capacity (TAC), total oxidant status (TOS) and the oxidative stress index (OSI) were compared between groups.

Results Subjects were randomly assigned to herbal tea $(n=40)$ and placebo $(n=40)$ groups. In first and second samples TAC, TOS and OSI were similar in both groups. TAC ( $p=0.375$ and $p=0.058)$ was lower and TOS ( $p=0.382$ and $p=0.118)$ was higher in second samples compared to first samples in both groups but not significantly. In second samples OSI ( $p=0.024$ and $p=0.007)$ increased significantly compared to first day in each group.

Conclusions Galactagogue herbal tea used in this study has no effect on global oxidant and antioxidant status of HM. Oxidative stress index increases in course of lactation compared to colostrum.

\section{INTRASUBJECT VARIATION IN ESTIMATES OF NEONATAL INTRAHEPATOCELLULAR LIPID USING 1H NMR SPECTROSCOPY}

